\title{
A Novel Parametric Controller Design Method for Hopf Bifurcation System
}

\author{
Jinbo Lu, Xiaorong Hou ${ }^{*}$ and Min Luo \\ School of Energy Science and Engineering, University of Electronic Science and \\ Technology of China, 611131, Chengdu, Sichuan, China \\ 564276721@qq.com,houxr@uestc.edu.cn,36205007@qq.com
}

\begin{abstract}
In this paper, using nonlinear state feedback, a novel general formula is proposed for the problem of Hopf bifurcation system control. This method does not increase dimensions of the system and keeps equilibria of the original system unchanged. The Rössler system is used as an example to illustrative the application of the method. Using Hurwitz criterion, the constraints of the parametric controller are derived. The idea of cylindrical algebraic decomposition (CAD) is employed to compute the constraints to find the parameter ranges of the designed controller, and the controller can be designed to stabilize the system using any feasible control parameters in the ranges. The designed controller not only can stabilize two non-symmetric equilibria but also can keep the Hopf bifurcations at the equilibria of the Rössler system. Computer simulation results are presented to demonstrate the theoretical results.
\end{abstract}

Keywords: Parametric controller, Hopf bifurcation control, Cylindrical Algebraic Decomposition (CAD), Rössler system

\section{Introduction}

In the Hopf bifurcation control, the basic task is to change the system dynamics in order to achieve the desired properties, such as delaying the onset of an inherent bifurcation, changing the parameter value of an existing bifurcation point, stabilizing a bifurcated branch, modifying the shape or type of a bifurcation chain, optimizing the system performance near a bifurcation point, or a combination of some of these objectives [1-2].

The technique of Hopf bifurcation control has been widely applied to solve physical and engineering problems (including electronic circuits [3-5], power systems [6-7], chemical [8], etc.). The control of Hopf bifurcation system has become a prime subject in nonlinear dynamics and has been widely investigated in recent years [9-12]. Many techniques of Hopf bifurcation control have been found. For example, in [10], a smallworld network model with the delay feedback is considered and with the controller, one can change the critical value of bifurcation and thus enlarge the stable region. Paper [11] presents and discusses a methodology to include in the power system security assessment and control functions a module to deal with Hopf Bifurcations. All these studies are effective, but the Hopf bifurcation problems and the designed controllers are special. In some research, the design methods of Hopf bifurcation control have been discussed [1316]. The designed controller better than before, but they are still limited to general applications. This fact motivates our work for the paper.

More notable is the works in [1,20], using parameterization technology and nonlinear state feedback, a general explicit formula is derived for controlling Hopf bifurcations. The formula, which can be applied to many kind of dynamic systems, and not change the equilibria of the original system. In [1], the method is applied to the Hopf bifurcation control of the Lorenz equation and Rössler system, and the computer simulation results 
are presented to confirm the analytical predictions. However, the controller designed by the method has its disadvantages: (i) the form is complex; (ii) the order is high; (iii) the controller parameter space has not been solved completely; (iv) the equilibria are included in the controller. In [20], the method in [1] has been improved, and the Rössler system is used as an illustrative example to present the new method. The form of designed controller is simplified, and the order is lower. The approach applies the graphical technique to find the stability boundaries of equilibrium points in parameter space, and then the parameters have been solved completely.

Although the controller form in [20] is simpler than which in [1], it is still complex and the equilibria are also included in the controller. In [21], the further application has been done, but the paper does not improve the controller. Therefore, in this paper a novel parametric controller design method is proposed for Hopf bifurcation system. The designed controller is composed of the system functions and the polynomial of system states, and keeps the equilibria of origin system unchanged. Not included the equilibrium point of the system, the formula is general and it is simpler than the one in [20]. The stability constraints of the controller parameters at equilibria are obtained using the Hurwitz criterion, and the complete parameters space of the controller can be found using the graphical technique (Cylindrical Algebraic Decomposition, CAD). The Rössler system is used as an example to illustrative the application of the approach.

In this work, the method of controller design for Hopf bifurcation systems and its proof is present in Section II. In Section III, using the Rössler system as an example, the process of the controller design is illustrated and the controller parameter space is solved. In Section IV, the computer simulation results are presented. Finally, the attained conclusions are presented in Section V.

\section{A General Function of Hopf Bifurcation System and Parametric Controller Design}

Before discussing the design of Hopf bifurcation control system, a general form for the system representation is presented. The proposed system description is not restricted to Hopf bifurcation, and it can be applied to study the control of other singularities or bifurcations such as zero bifurcations. To be more specific, consider the following general nonlinear system:

$$
\dot{X}=F(X, \mu)
$$

Where the dot denotes differentiation with respect to time $t, X=\left(x_{1}, x_{2}, \cdots, x_{n}\right)^{T}$ is a $n$ dimensional state vector, while $\mu \in R$ is a scalar parameter, called bifurcation parameter (Note, generally, one may assume that ${ }^{\mu}$ is an $m$-dimensional vector for $m \geq 1$.). $F(X, \mu)=\left[f_{1}(X, \mu), f_{2}(X, \mu), \cdots, f_{n}(X, \mu)\right]^{T}$ is a matrix of nonlinear polynomial system that has Hopf bifurcations. Let $X^{*}$ be an equilibrium of the system, i.e. $F\left(X^{*}, \mu\right) \equiv 0$, for any value of $\mu$.

In this paper, we design a Hopf bifurcation controller for the 3 dimensional system, then consider the following theorem.

Theorem 1: A 3 dimension system can be considered as:

$$
\left\{\begin{array}{l}
\dot{x}_{1}=f_{1}(X, \mu) \\
\dot{x}_{2}=f_{2}(X, \mu) \\
\dot{x}_{3}=f_{3}(X, \mu)
\end{array}\right.
$$

where, $X=\left[x_{1}, x_{2}, x_{3}\right]^{T}$. Let $\operatorname{det}(J) \neq 0, J$ is the Jacobian at equilibrium $X^{*}$ of system (2). The parametric controller can be designed as: 


$$
u(X, a)=\left[u_{1}(X, a), u_{2}(X, a), u_{3}(X, a)\right]^{T},
$$

Generally, only one of $u_{1}(X, a), u_{2}(X, a)$ and $u_{3}(X, a)$ need to be designed, the others will be choose to zero. Here, the control can be written as:

$$
u_{j}(X, a)=g(X, a) f_{j}(X, a)+\sum_{k=1}^{n} c_{0 k} f_{k}(X, a)
$$

where $j=1,2,3, k=1,2,3, k \neq j, u_{j}(X, a)$ is composed of the system vector basis and

$$
g(X, a)=c_{1}+c_{2} x_{1}+c_{3} x_{2}+\cdots+c_{n+1} x_{n}+c_{n+2} x_{1}^{2}+c_{n+3} x_{2}^{2}+\cdots+c_{2 n+1} x_{n}^{2}
$$

$\left\{c_{0 k}, c_{1}, c_{2}, \ldots, c_{2 n+1} \in R\right\}, n=3$ are the parameters of the designed controller. If the controller parameters can satisfy the constraints of the Hurwitz criterion, the system (2) can be controlled by selecting the controller parameter values.

Proof:

To be more simplifying, generally, the parametric controller can be designed as:

$$
u_{2}(X, \mu)=g(X) \cdot f_{2}(X, \mu)+c_{01} f_{1}(X, \mu)
$$

here, $g(X)=\left(c_{1}+c_{2} x_{1}+c_{3} x_{2}^{2}+c_{4} x_{3}^{2}\right),\left\{c_{01}, c_{1}, c_{2}, c_{3}, c_{4} \in R\right\}$, and $c_{01}, c_{1}, c_{2}, c_{3}, c_{4}$ are controller parameters.

Add the controller (5), the system can be rewritten as:

$$
\left\{\begin{array}{l}
\dot{x}_{1}=f_{1}(X, \mu) \\
\dot{x}_{2}=f_{2}(X, \mu)+u_{2}(X, \mu) \\
\dot{x}_{3}=f_{3}(X, \mu)
\end{array}\right.
$$

for this closed loop system, the Jacobian is given as:

where,

$$
J=\left[\begin{array}{lll}
\frac{\partial f_{1}}{\partial x_{1}} & \frac{\partial f_{1}}{\partial x_{2}} & \frac{\partial f_{1}}{\partial x_{3}} \\
j_{21} & j_{22} & j_{23} \\
\frac{\partial f_{3}}{\partial x_{1}} & \frac{\partial f_{3}}{\partial x_{2}} & \frac{\partial f_{3}}{\partial x_{3}}
\end{array}\right]
$$

$$
\begin{gathered}
j_{21}=\frac{\partial f_{2}}{\partial x_{1}}+\frac{\partial f_{2}}{\partial x_{1}} g(X)+c_{2} f_{2}+c_{01} \frac{\partial f_{1}}{\partial x_{1}} \\
j_{22}=\frac{\partial f_{2}}{\partial x_{2}}+\frac{\partial f_{2}}{\partial x_{2}} g(X, \mu)+2 c_{3} f_{2} x_{2}+c_{01} \frac{\partial f_{1}}{\partial x_{2}} \\
j_{23}=\frac{\partial f_{2}}{\partial x_{3}}+\frac{\partial f_{2}}{\partial x_{3}} g(X, \mu)+2 c_{4} f_{2} x_{3}+c_{01} \frac{\partial f_{1}}{\partial x_{3}}
\end{gathered}
$$

Let the system equilibrium is $X_{0}=\left(x_{10}, x_{20}, x_{30}\right)$, then the system characteristic equation at the equilibrium can be given as:

$$
G(\lambda)=\operatorname{det}(\lambda I-J)=a_{0} \lambda^{3}+a_{1} \lambda^{2}+a_{2} \lambda+a_{3}
$$

where,

$$
\begin{gathered}
a_{0}=1 \\
a_{1}=\left[-c_{01} \frac{\partial f_{1}}{\partial x_{2}}-\frac{\partial f_{1}}{\partial x_{1}}-\frac{\partial f_{2}}{\partial x_{2}}-\frac{\partial f_{2}}{\partial x_{2}} g(X, \mu)-\frac{\partial f_{3}}{\partial x_{3}}-2 c_{3} f_{2} x_{2}\right]_{X=X_{0}}
\end{gathered}
$$




$$
\begin{aligned}
& a_{2}=\left[\left(\frac{\partial f_{1}}{\partial x_{1}} \frac{\partial f_{2}}{\partial x_{2}}-\frac{\partial f_{1}}{\partial x_{2}} \frac{\partial f_{2}}{\partial x_{1}}-\frac{\partial f_{2}}{\partial x_{3}} \frac{\partial f_{3}}{\partial x_{2}}+\frac{\partial f_{2}}{\partial x_{2}} \frac{\partial f_{3}}{\partial x_{3}}\right) g(X, \mu)-c_{2} \frac{\partial f_{1}}{\partial x_{2}} f_{2}-2 c_{4} x_{3} \frac{\partial f_{3}}{\partial x_{2}} f_{2}\right. \\
& +2 c_{3} f_{2}\left(\frac{\partial f_{3}}{\partial x_{3}}+\frac{\partial f_{1}}{\partial x_{1}}\right) x_{2}+c_{01}\left(\frac{\partial f_{3}}{\partial x_{3}} \frac{\partial f_{1}}{\partial x_{2}}-\frac{\partial f_{1}}{\partial x_{3}} \frac{\partial f_{3}}{\partial x_{2}}\right)+\frac{\partial f_{1}}{\partial x_{1}} \frac{\partial f_{2}}{\partial x_{2}}+\frac{\partial f_{1}}{\partial x_{1}} \frac{\partial f_{3}}{\partial x_{3}}-\frac{\partial f_{1}}{\partial x_{2}} \frac{\partial f_{2}}{\partial x_{1}} \\
& \left.-\frac{\partial f_{1}}{\partial x_{3}} \frac{\partial f_{3}}{\partial x_{1}}-\frac{\partial f_{2}}{\partial x_{3}} \frac{\partial f_{3}}{\partial x_{2}}+\frac{\partial f_{2}}{\partial x_{2}} \frac{\partial f_{3}}{\partial x_{3}}\right]_{X=X_{0}} \\
& a_{3}=\left[\left(-\frac{\partial f_{1}}{\partial x_{1}} \frac{\partial f_{2}}{\partial x_{2}} \frac{\partial f_{3}}{\partial x_{3}}+\frac{\partial f_{1}}{\partial x_{1}} \frac{\partial f_{2}}{\partial x_{3}} \frac{\partial f_{3}}{\partial x_{2}}+\frac{\partial f_{1}}{\partial x_{2}} \frac{\partial f_{2}}{\partial x_{1}} \frac{\partial f_{3}}{\partial x_{3}}-\frac{\partial f_{1}}{\partial x_{2}} \frac{\partial f_{2}}{\partial x_{3}} \frac{\partial f_{3}}{\partial x_{1}}-\frac{\partial f_{1}}{\partial x_{3}} \frac{\partial f_{2}}{\partial x_{1}} \frac{\partial f_{3}}{\partial x_{2}}\right.\right. \\
& \left.+\frac{\partial f_{1}}{\partial x_{3}} \frac{\partial f_{2}}{\partial x_{2}} \frac{\partial f_{3}}{\partial x_{1}}\right) g(X, \mu)+\left(-2 c_{3} \frac{\partial f_{1}}{\partial x_{1}} \frac{\partial f_{3}}{\partial x_{3}} x_{2}+2 c_{4} \frac{\partial f_{1}}{\partial x_{1}} \frac{\partial f_{3}}{\partial x_{2}} x_{3}-2 c_{4} \frac{\partial f_{1}}{\partial x_{2}} \frac{\partial f_{3}}{\partial x_{1}} x_{3}+c_{2} \frac{\partial f_{1}}{\partial x_{2}} \frac{\partial f_{3}}{\partial x_{3}}\right. \\
& \left.+2 c_{3} \frac{\partial f_{1}}{\partial x_{3}} \frac{\partial f_{3}}{\partial x_{1}} x_{2}-c_{2} \frac{\partial f_{1}}{\partial x_{3}} \frac{\partial f_{3}}{\partial x_{2}}\right) f_{2}-\frac{\partial f_{1}}{\partial x_{1}} \frac{\partial f_{2}}{\partial x_{2}} \frac{\partial f_{3}}{\partial x_{3}}+\frac{\partial f_{1}}{\partial x_{1}} \frac{\partial f_{2}}{\partial x_{3}} \frac{\partial f_{3}}{\partial x_{2}}+\frac{\partial f_{1}}{\partial x_{2}} \frac{\partial f_{2}}{\partial x_{1}} \frac{\partial f_{3}}{\partial x_{3}} \\
& \left.-\frac{\partial f_{1}}{\partial x_{2}} \frac{\partial f_{2}}{\partial x_{3}} \frac{\partial f_{3}}{\partial x_{1}}-\frac{\partial f_{1}}{\partial x_{3}} \frac{\partial f_{2}}{\partial x_{1}} \frac{\partial f_{3}}{\partial x_{2}}+\frac{\partial f_{1}}{\partial x_{3}} \frac{\partial f_{2}}{\partial x_{2}} \frac{\partial f_{3}}{\partial x_{1}}\right]_{X=X_{0}}
\end{aligned}
$$

here, $f_{i}=f_{i}\left(x_{1}, x_{2}, x_{3}\right), i=1,2,3$.

It can be seen, when $X=X_{0}$, the values of $\frac{\partial f_{i}}{\partial x_{j}}$ and $f_{i}$ are fixed, here $i=1,2,3$, $j=1,2,3$. Therefore, values and symbols of $a_{1}, a_{2}$ and $a_{3}$ can be changed by $c_{01}, c_{1}, c_{2}$, $c_{3}, c_{4}$. According to the Hurwitz criterion, the constraints of the controller parameters can be written as the following:

$$
\sum:\left\{\begin{array}{l}
a_{1}>0 \\
a_{2}>0 \\
a_{3}>0 \\
g \geq 0
\end{array}\right.
$$

here, $g=a_{1} a_{2}-a_{3}$.

So, if the constraints $\Sigma$ can be satisfied, the system (2) can be controlled by selecting the values of controller parameter $c_{01}, c_{1}, c_{2}, c_{3}$ and $c_{4}$.

Remarks: By no means the control formula given in Eq. (3) and Eq. (4) are a unique control law. There are many other feasible controllers.

As shown in the next section, as an example, the Rössler system will be applied to illustrate the effectiveness of the controller designed in Theorem 1.

\section{An Example}

The Rössler system can exhibit complex dynamics, including equilibria, limit cycles, and chaos. In this section, we will apply it as an example to demonstrate the calculation method of designed controller parameters and to prove the effectiveness of the controller. 


\subsection{Rössler System Description}

The Rössler system can be described as

$$
\left\{\begin{array}{l}
\dot{x}=-y-z \\
\dot{y}=x+a y \\
\dot{z}=b+z(x-c)
\end{array}\right.
$$

where $X=[x, y, z]^{T}, a, b, c$ are adjustable parameters, and. Here, $a$ is selected as bifurcation parameter. We suppose that $\left(x_{0}, y_{0}, z_{0}\right)$ is an equilibrium of the system (7), then $y_{0}=-z_{0}, x_{0}=a z_{0}$.

\subsection{Controller Design}

According to equation (3), the controller of the Rössler system is designed as:

$$
u_{2}(X, a)=g(X) \cdot f_{2}(X, a)+c_{01} f_{1}(X, a),
$$

where

$$
g(X)=\left(c_{1}+c_{2} x+c_{3} y^{2}+c_{4} z^{2}\right)
$$

and $c_{01}, c_{1}, c_{2}, c_{3}, c_{4}$ are the parameters of the controller. $c_{01} \in R, c_{1} \in R, c_{2} \in R$, $c_{3} \in R, c_{4} \in R$. To be more simplifying, here $c_{01}=c_{0}, c_{3}=c_{4}=0$ are considered, then $u_{2}(X, a)$ can be rewritten as:

$$
u_{2}(X, a)=\left(c_{1}+c_{2} x\right)(x+a y)+c_{0}(-y-z)
$$

The order of the designed controller $(8)$ is 2 . It is easy to see that the order in this paper is lower than the one in [20], where it is 3.

Adding the controller $u_{2}(X, a)$ to original system (7), the new system with nonlinear state feedback controls is:

$$
\left\{\begin{array}{l}
\dot{x}=-y-z \\
\dot{y}=x+a y+u_{2}(X, a) \\
\dot{z}=b+z(x-c)
\end{array}\right.
$$

The controller (8) does not change the equilibria of the original system (7). In order to calculate the parameters of $u_{2}(X, a)$, evaluating the Jacobian of system (9) at the equilibrium yields:

$$
J_{E}=\left[\begin{array}{ccc}
0 & -1 & -1 \\
1+c_{1}+c_{2} a z_{0} & a+a c_{1}+c_{2} a^{2} z_{0}-c_{0} & -c_{0} \\
z_{0} & 0 & a z_{0}-c
\end{array}\right]
$$

which gives the characteristic polynomial :

where

$$
G(\lambda)=\operatorname{det}\left(\lambda I-J_{E}\right)=\lambda^{3}+a_{1} \lambda^{2}+a_{2} \lambda+a_{3}
$$

$$
\begin{gathered}
a_{1}=c-c_{2} a^{2} z_{0}-a-a c_{1}-a z_{0}+c_{0} \\
a_{2}=c_{1}+c_{2} a z_{0}-c_{2} a^{2} b-c a c_{1}+a^{2} z_{0}+c c_{0}+1+a^{2} z_{0} c_{1}-a z_{0} c_{0}+z_{0}-a c \\
a_{3}=2 c_{2} a b-2 a z_{0}+c+c c_{1}-c c_{2} a z_{0}-2 z_{0} a c_{1}
\end{gathered}
$$

Using the controller parameter constraints (6), the stability conditions of the equilibria are

and

$$
a_{j}>0, j=1,2,3
$$




$$
g \geq 0
$$

The equation (13) must be satisfied, which imply that the static bifurcation does not exist in controlled Rössler system. In equation (14), $g>0$ is a system stability condition, at $g=0$ Hopf bifurcation occurs.

\subsection{System Constraints Calculation}

The Rössler system trajectories change significantly at various system parameters, considering the system parameters as $a=2 / 5, b=2, c=4$. While without the controller in the system, the trajectory is in chaotic state at the equilibrium. In the following, the numerical results of the constraints based on the inequalities (13) and (14) are calculated as:

$$
\begin{gathered}
a_{1}=c_{0}+\frac{18}{5}-\frac{2}{5} c_{1}-\frac{4}{25} c_{2} z_{0}-\frac{2}{5} z_{0} \\
a_{2}=4 c_{0}-\frac{2}{5} c_{0} z_{0}-\frac{3}{5} c_{1}+\frac{4}{25} c_{1} z_{0}-\frac{8}{25} c_{2}+\frac{2}{5} c_{2} z_{0}+\frac{29}{25} z_{0}-\frac{3}{5} \\
a_{1}=4 c_{1}-\frac{4}{5} c_{1} z_{0}+\frac{8}{5} c_{2}-\frac{8}{5} c_{2} z_{0}-\frac{4}{5} z_{0}+4
\end{gathered}
$$

From system (7), the equilibria are$$
E_{e}^{-}=\left(2-\frac{4}{5} \sqrt{5},-(5-2 \sqrt{5}), 5-2 \sqrt{5}\right)
$$$$
\approx(0.211145618,-0.527864045,0.527864045)
$$$$
E_{e}^{+}=\left(2+\frac{4}{5} \sqrt{5},-(5+2 \sqrt{5}), 5+2 \sqrt{5}\right)
$$$$
\approx(3.788854382,-9.472135955,9.472135955)
$$

Firstly, to satisfy the constraints in (13) and (14), the Hurwitz criterion parameters at equilibrium $E_{e}^{-}$are

$$
\begin{gathered}
a_{1}^{-}=c_{0}-\frac{2}{5} c_{1}-\frac{4}{25}(5-2 \sqrt{5}) c_{2}+\frac{8}{5}+\frac{4}{5} \sqrt{5} \\
\approx c_{0}-0.4 c_{1}-0.08445824720 c_{2}+3.38854382 \\
a_{2}^{-}=\left(2+\frac{4}{5} \sqrt{5}\right) c_{0}+\left(\frac{1}{5}-\frac{8}{25} \sqrt{5}\right) c_{1}+\left(\frac{42}{25}-\frac{4}{5} \sqrt{5}\right) c_{2}+\frac{26}{5}-\frac{58}{25} \sqrt{5} \\
\approx 3.788854382 c_{0}-0.5155417528 c_{1}-0.1088543820 c_{2}+0.0123222922 \\
a_{3}^{-}=\frac{8}{5} \sqrt{5} c_{1}+\left(-\frac{32}{5}+\frac{16}{5} \sqrt{5}\right) c_{2}+\frac{8}{5} \sqrt{5} \\
\approx 3.577708764 c_{1}+0.7554175280 c_{2}+3.577708764 \\
g^{-}=a_{1}^{-} a_{2}^{-}-a_{3}^{-} \quad 0.515541753 c_{0}^{2}+0.08248668045 c_{1}^{2}+0.0036774601 c_{2}^{2}-0.8124334022 c_{0} c_{1} \\
-0.1715417528 c_{0} c_{2}+0.03483340224 c_{1} c_{2}+5.140879227 c_{0}-2.131893444 c_{1} \\
-0.45013995857 c_{2}-1.414380124
\end{gathered}
$$

Secondly, the Hurwitz criterion parameters at equilibrium $E_{e}^{+}$are

$$
\begin{aligned}
a_{1}^{+} & =c_{0}-\frac{2}{5} c_{1}-\frac{4}{25}(5+2 \sqrt{5}) c_{2}+\frac{8}{5}-\frac{4}{5} \sqrt{5} \\
& \approx c_{0}-0.4 c_{1}-1.515541753 c_{2}-0.188854382
\end{aligned}
$$




$$
\begin{aligned}
& a_{2}^{+}=\left(2-\frac{4}{5} \sqrt{5}\right) c_{0}+\left(\frac{1}{5}+\frac{8}{25} \sqrt{5}\right) c_{1}+\left(\frac{42}{25}+\frac{4}{5} \sqrt{5}\right) c_{2}+\frac{26}{5}+\frac{58}{25} \sqrt{5} \\
& \approx 0.211145618 c_{0}+0.9155417530 c_{1}+3.468854382 c_{2}+10.38767771 \\
& a_{3}^{+}=-\frac{8}{5} \sqrt{5} c_{1}+\left(-\frac{32}{5}-\frac{16}{5} \sqrt{5}\right) c_{2}-\frac{8}{5} \sqrt{5} \\
& \approx-3.577708764 c_{1}-13.55541753 c_{2}-3.577708764 \\
& g^{+}=a_{1}^{+} a_{2}^{+}-a_{3}^{+} \\
& \approx 0.084458247 c_{0}^{2}-0.1464866804 c_{1}^{2}-2.10287746 c_{2}^{2}+0.332433402 c_{0} c_{1} \\
& +1.259541753 c_{0} c_{2}-1.110033402 c_{1} c_{2}+4.139120773 c_{0}-0.300106556 c_{1} \\
& -1.137060041 c_{2}+0.646380124
\end{aligned}
$$

Then the constraints of the system from (13) can be given as:

$$
\left\{\begin{array}{l}
a_{j}^{-}>0 \\
a_{j}^{+}>0, j=1,2,3 ;
\end{array}\right.
$$

It can be noted that if $c_{2}=0 ; a_{3}^{-}>0$ and $a_{3}^{+}>0$ will never occur at same time. Therefore, the condition of $c_{2} \neq 0$ must be satisfied for the given design. From $a_{3}^{-}>0$ and $a_{3}^{+}>0$, following inequalities can be obtained:

$$
\begin{gathered}
3.577708764 c_{1}+0.7554175280 c_{2}+3.577708764>0 \text { and } \\
-3.577708764 c_{1}-13.55541753 c_{2}-3.577708764>0 .
\end{gathered}
$$

These can be rewritten as:

$$
\left\{\begin{array}{l}
c_{1}>-1.0-0.2111456180 c_{2} \\
c_{1}<-1.0-3.788854383 c_{2}
\end{array}\right.
$$

here, if $c_{2}>=0$, the two inequalities cannot be satisfied at same time. Therefore, $c_{2}<0$ and $c_{1}>-1$.

It is noted that, from the Cylindrical Algebraic Decomposition (CAD) theory, the problem of solving the controller parameters $c_{1}, c_{2}$ and $c_{3}$ can be transformed into threedimensional space segmentation problem, and their characteristic polynomials are the dividing planes of the space. The divided sub-space is continuous and the characteristics of all points are consistent; therefore, a feature of any point in the subspace can explain the subspace features. The basic range of ${ }^{c_{1}}$ and ${ }^{c_{2}}$ have been identified in (16). In order to simplify the process, $c_{2}=-2$ is chosen in this case. So the three-dimensional segmentation problem is converted into a two-dimensional space segmentation problem.

Then conditions can be simplified to

$$
\begin{gathered}
a_{1}^{-}=c_{0}-0.4 c_{1}+3.557770876 \\
a_{2}^{-}=3.788854382 c_{0}-0.5155417528 c_{1}+0.2300310562 \\
a_{3}^{-}=3.577708764 c_{1}+2.066873708 \\
g^{-}=1.515541753 c_{0}^{2}+0.08248668045 c_{1}^{2}-0.8124334022 c_{0} c_{1} \\
+5.483962733 c_{0}-2.201560248 c_{1}-0.4993903666 \\
a_{1}^{+}=c_{0}-0.4 c_{1}+2.842229124 \\
a_{2}^{+}=0.211145618 c_{0}+0.9155417530 c_{1}+3.449968946
\end{gathered}
$$




$$
\begin{gathered}
a_{3}^{+}=-3.577708764 c_{1}+23.53312630 \\
g^{+}=0.084458247 c_{0}^{2}-0.1464866804 c_{1}^{2}+0.3324334020 c_{0} c_{1} \\
+1.620037267 c_{0}+1.919960248 c_{1}-5.491009634
\end{gathered}
$$

The stability boundaries of the system for the designed controller can be obtained from (17) (24).

\subsection{Calculation of Parameters Using CAD}

In general, it is difficult to calculate the parameters fully from the conditions provided in equations (17) to (24). CAD is suitable for parameter range solving problems, and has been applied to the system design [17]. In this paper, CAD is used to solve the range of parameters $c_{0}$ and $c_{1}$. As $c_{2}=-2$ is selected, in the designed controller there are two parameters ( $c_{0}$ and ${ }^{c_{1}}$ ) that need to be calculated. A set of curves for the conditions (17) to (24) has been drawn on the $c_{0}-c_{1}$ plane in Figure 1. Thereafter, the regions where the Rössler system can be controlled are extracted. The parameters $c_{0}$ and $c_{1}$ in each region are examined to identify the set of values that satisfy the criteria. Finally, region $Q$ is found, which is surrounded by $a_{3}^{-}>0, g^{-}>0, a_{3}^{+}>0, g^{+}>0$. $L_{1}$ is part of the curves of $g^{-}$(from point $A$ to $B$ ) and $L_{2}$ is part of the curves of $g^{+}$(from point $B$ to $C$ ).

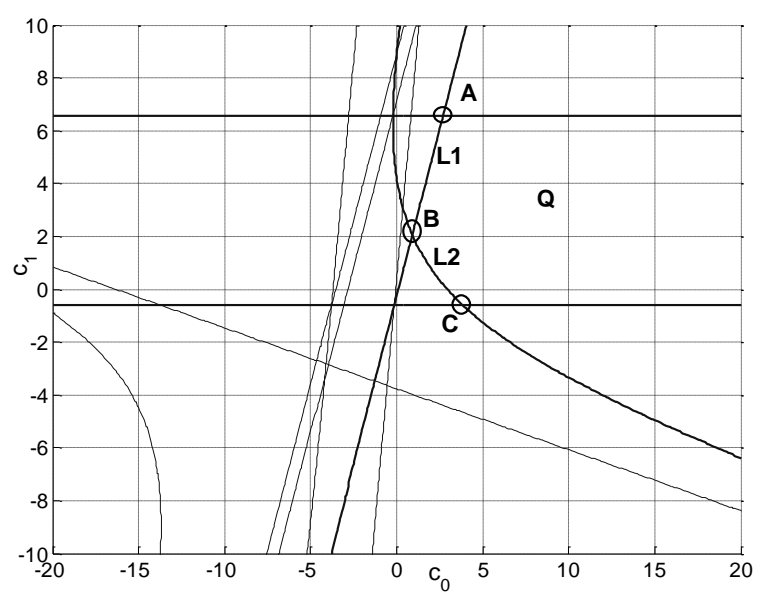

\section{Figure 1. System Constraints Curves in $c_{0}-c_{1}$ Plane}

Point $A, B$, and $C$ are three specific points on $c_{0}-c_{1}$ plane, where $A$ is the crossover point of conditions (20) and (23). From (20) and (23), $c_{1}=6.577708766$, and $c_{0}=2.698244265$ are obtained. $B$ is the crossover point of conditions (20) and (24). According to the two equations, the coordinate of $B$ can be obtained, and these are found as $c_{1}=2.061683145$ and $c_{0}=0.9048934015 . C$ is the crossover point of condition (19) and (24), and the values of parameters corresponding to this point are $c_{1}=-0.5777087640, c_{0}=3.801520372$. 


\section{The Simulations}

In this section, the parameters of the controller are calculated based on different cases of the system and the simulations will be done. The states of the selected Rössler system are in chaos state at the equilibria without the application of controller. After the addition of controller, the system states can be controlled within a stable range. There are four cases of dynamic situations near the two equilibria $E_{e}^{-}$and $E_{e}^{+}$: (i) both the equilibria become stable without Hopf bifurcations; (ii) $E_{e}^{+}$is stable while $E_{e}^{-}$has a supercritical Hopf bifurcation; (iii) $E_{e}^{-}$is stable while $E_{e}^{+}$has a supercritical Hopf bifurcation; and (iv) both equilibria have Hopf bifurcations.

In order to ensure that there is no bifurcation in all of the four cases, the conditions $a_{i}^{-}>0$ and $a_{i}^{+}>0$ must be satisfied. The four cases are decided by $g^{-}$and $g^{+}$. In fact, if $g^{-}<0$ the system cannot be stabilized at $E_{e}^{-}$, and if $g^{+}<0$ the system cannot be stabilized at $E_{e}^{+}$. When $g^{-}=0$, the system trajectory at $E_{e}^{-}$is Hopf bifurcation, and limit cycle is stable. Similarly at $E_{e}^{+}$the trajectory is Hopf bifurcation when $g^{+}=0$.

In the following, the values of $c_{0}$ and $c_{1}$ will defined for each of the four cases.

Case (i): Both equilibria become stable without Hopf bifurcations. Both equilibria $E_{e}^{-}$ and $E_{e}^{+}$of the controlled system (9) are stable and no bifurcations occur. Then the following inequalities must be satisfied.

$$
\left\{\begin{array}{l}
g^{-}>0 \\
g^{+}>0
\end{array}\right.
$$

For this case $c_{0}$ and ${ }^{c_{1}}$ should be selected in $Q$ only, avoiding any boundary. Here these are selected as $c_{0}=1.5, c_{1}=2$ for simulation.

Case (ii): $E_{e}^{+}$is stable while $E_{e}^{-}$has a Hopf bifurcation. For this case, following constraints must be satisfied:

$$
\left\{\begin{array}{l}
g^{-}=0 \\
g^{+}>0
\end{array}\right.
$$

Here $c_{0}$ and $c_{1}$ should be selected on the line ${ }^{L_{1}}$ but it should not include $A$ or $B$. Hence, $c_{0}=1.5$ is selected, and $c_{1}=3.562109985$ is obtained from (20) and (24).

Case (iii): $E_{e}^{-}$is stable while $E_{e}^{+}$has a Hopf bifurcation. Similar to the Case (ii), the following conditions should be satisfied.

$$
\left\{\begin{array}{l}
g^{-}>0 \\
g^{+}=0
\end{array}\right.
$$

Where $c_{0}$ and ${ }^{c_{1}}$ should be selected on the line $L_{2}$ but it must not include $B$ or $C$. Hence, $c_{0}=1.5$ is selected, and $c_{1}=1.287395373$.

Case (iv): Both equilibria have Hopf bifurcations. Following condition must be satisfied.

$$
\left\{\begin{array}{l}
g^{-}=0 \\
g^{+}=0
\end{array}\right.
$$


This case only be satisfied at the crossover point of the curves $g^{-}=0$ and $g^{+}=0$, where it is the point $B$ in Figure 1 , resulting in $c_{0}=0.9048934015$ and $c_{1}=2.061683145$. At this case, the controller can keep the Hopf bifurcations from the equilibria of the Rössler system.

Then the controller parameters have been calculated for all the four cases. Simulations have been performed in order to verify the effectiveness of the controller, and numerical results for the four cases are shown in Figures 2 5, where $a=2 / 5, b=2, c=4$.

Figures 2(a) and 2(b) show that both $E_{e}^{-}$and $E_{e}^{+}$are stable when the conditions given in (41) are satisfied [Case (i)]. Where the selected controller parameter values are $c_{0}=1.5 \quad, \quad c_{1}=2, \quad c_{2}=-2$; with $\left(x_{0}, y_{0}, z_{0}\right)=(0.3,0.5,0.7)$ for $\quad(a), \quad$ and $\left(x_{0}, y_{0}, z_{0}\right)=(3.5,-9.5,11.0)$ for $(\mathrm{b})$.

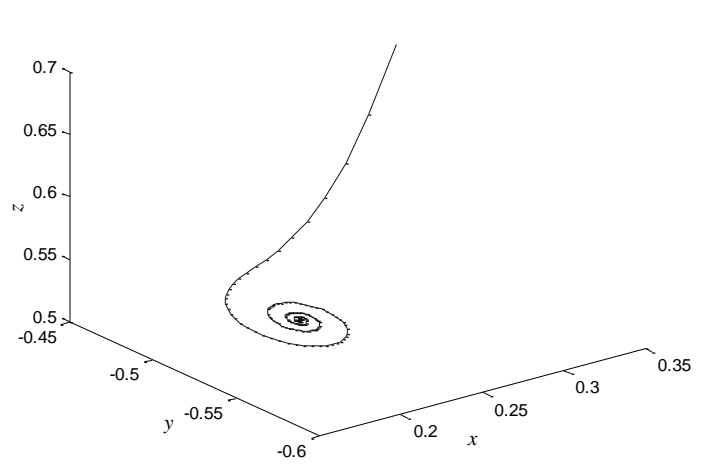

(a) Trajectory Near $E_{e}^{-}$

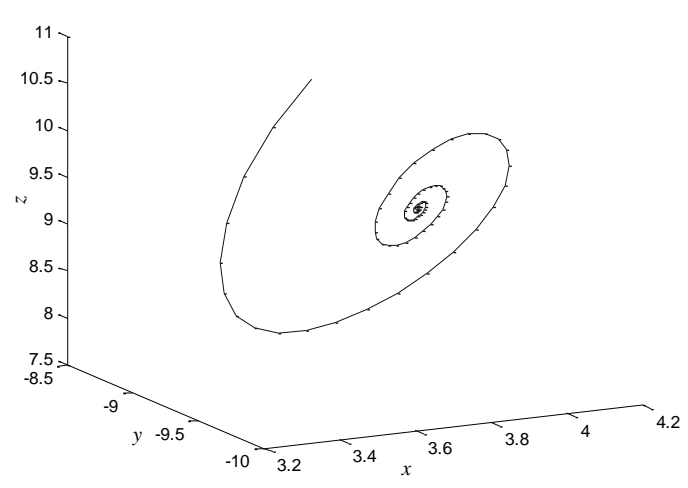

(b) Trajectory Near $E_{e}^{+}$

Figure 2. Trajectories of The Rössler System (12) when both $E_{e}^{-}$and $E_{e}^{+}$are Stable

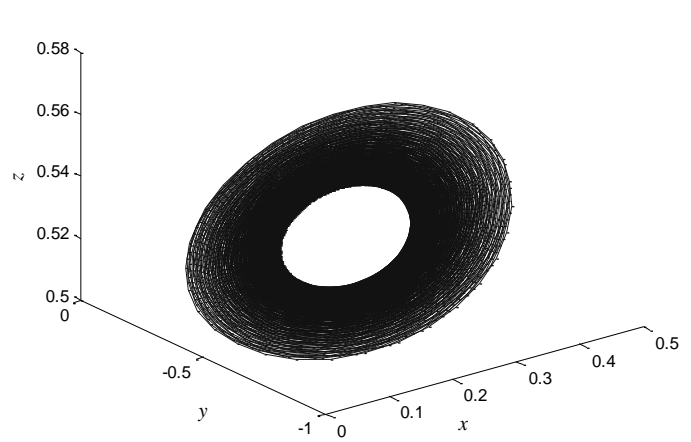

(a) Trajectory Near $E_{e}^{-}$

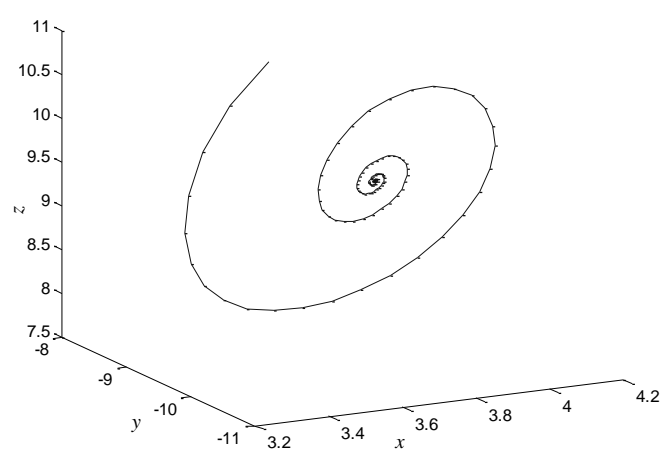

(b) Trajectory Near $E_{e}^{+}$

Figure 3. Trajectories of The Rössler System (12) when $E_{e}^{-}$is Hopf Bifurcation and $E_{e}^{+}$is Stable 


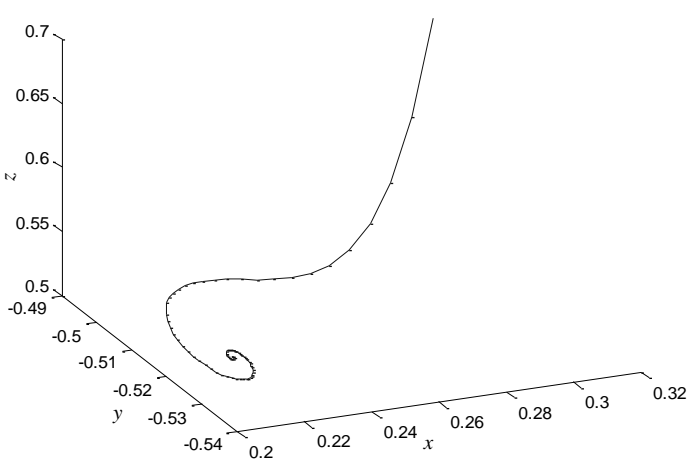

(a) Trajectory Near $E_{e}^{-}$

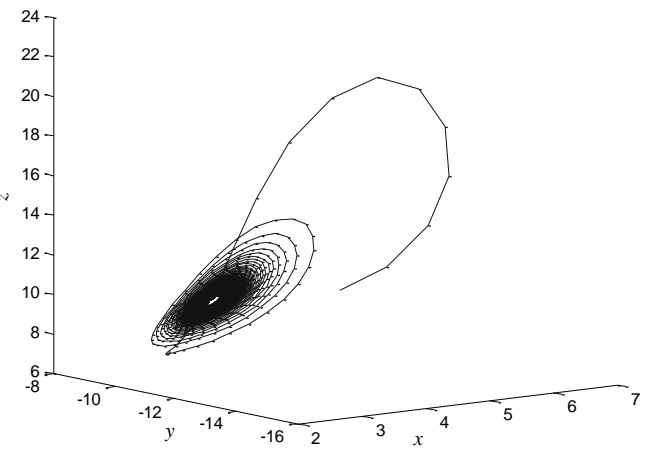

(b) Trajectory Near $E_{e}^{+}$

\section{Figure 4. Trajectories of The Rössler System (12) when $E_{e}^{+}$is Hopf}

\section{Bifurcation and $E_{e}^{-}$is Stable}

Figure 3(a) shows a stable limit cycle bifurcating from $E_{e}^{-}$, while 3(b) shows that $E_{e}^{+}$ is still stable [Case (ii)]. Where the selected controller parameter values are $c_{0}=1.5$, $c_{1}=3.562109985 \quad, \quad c_{2}=-2 \quad ; \quad$ with $\quad\left(x_{0}, y_{0}, z_{0}\right)=(0.4,0.5,0.5) \quad$ for $\quad(a), \quad$ and $\left(x_{0}, y_{0}, z_{0}\right)=(3.5,-9.5,11.0)$ for (b).

Figure 4(a) shows $E_{e}^{-}$is stable, and 4(b) shows a stable limit cycle bifurcating from $E_{e}^{+} \quad$ Case (iii)]. Where the selected controller parameter values are $c_{0}=1.5$, $c_{1}=1.287395373 \quad, \quad c_{2}=-2 \quad$; with $\left(x_{0}, y_{0}, z_{0}\right)=(0.3,0.5,0.7)$ for $\quad$ (a), and $\left(x_{0}, y_{0}, z_{0}\right)=(6.0,-9.0,9.0)$ for (b).

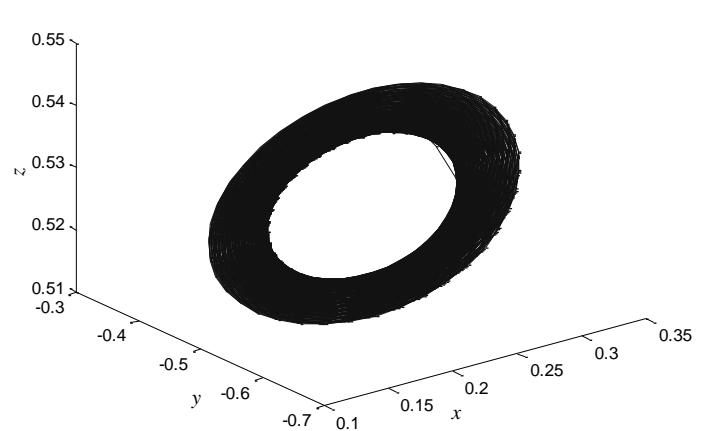

(a) Trajectory Near $E_{e}^{-}$

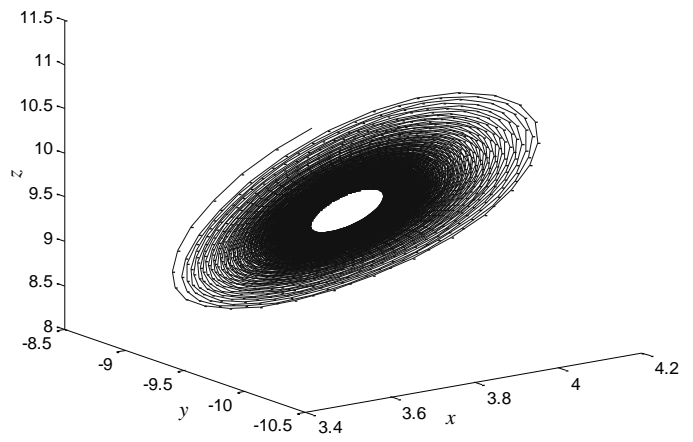

(b) Trajectory Near $E_{e}^{+}$

\section{Figure 5. Trajectories of The Rössler System (12) when both $E_{e}^{-}$and $E_{e}^{+}$are Hopf Bifurcations}

Figures 5(a) and 5(b) show that both $E_{e}^{-}$and $E_{e}^{+}$are unstable [Case (iv)], and they are surrounded by stable limit cycles. Where the selected controller parameter values are $c_{0}=0.9048934015, c_{1}=2.061683145, c_{2}=-2$; with $\left(x_{0}, y_{0}, z_{0}\right)=(0.3,-0.52,0.52)$ for (a), and $\left(x_{0}, y_{0}, z_{0}\right)=(3.7,-9.5,10.5)$ for (b). 


\section{Conclusion}

In this paper a Hopf bifurcation controller design method is proposed for nonlinear systems. The designed controller does not affect the equilibrium of the original system. As an example, the Rössler system is used to demonstrate the controller design and parameter solving process of the proposed method. The range of the controller parameters is established using CAD. Furthermore, four set of parameter values are given for different cases of Hopf bifurcation at two equilibria of the system. The analytical predictions have been verified by numerical simulation. Results show that the proposed controller design method be effectively applied to stabilize the system states. When the system parameters are same, compared to the existing works, the form of the designed controller in this paper is simpler and the order lower.

\section{Acknowledgments}

This work is supported by the National Natural Science Foundation of China (Grant Nos. 61374001 and 61074189).

\section{References}

[1] Y. Pei and G. Chen, "Hopf bifurcation control using nonlinear feedback with polynomial functions", International Journal of Bifurcation and Chaos, vol. 14, no. 5, (2004), pp. 1683-1704.

[2] G. Chen, J. Moiola and H. Wang, "Bifurcation control: Theories, Methods, and Applications", International Journal of Bifurcation and Chaos, vol. 10, no. 3, (2000), pp. 511-548.

[3] P. Deivasundari, G. Uma and R. Santhi, "Experimental verification of Hopf bifurcation in pulse-width modulated inverter fed cage induction motor drive system", IET Power Electronics, vol. 7, (2014), pp. 340-349.

[4] A. Mees and L. Chua, "The Hopf bifurcation theorem and its applications to nonlinear oscillations in circuits and systems", IEEE Transactions on Circuits and Systems, vol. 26, no. 4, (1979), pp. 235-254.

[5] A. Kavitha and G. Uma, "Experimental Verification of Hopf Bifurcation in DC-DC Luo Converter", IEEE Transactions on Power Electronics, vol. 23, no. 6, (2008), pp. 1-6.

[6] M. Huang, C. Tse, S. Wong, C. Wan and X. Ruan, "Low-Frequency Hopf Bifurcation and Its Effects on Stability Margin in Three-Phase PFC Power Supplies Connected to Non-Ideal Power Grid", IEEE Transactions on Circuits and System, vol. 60, no. 12, (2013), pp. 3328-3340.

[7] M. Tomim, B. Lopes, R. Leme, R. Jovita and A. Souza, "Modified Hopf bifurcation index for power system stability assessment", IET Generation, Transmission \& Distribution, vol. 152, no. 6, (2005), pp. 906-912.

[8] W. Gan, C. Tian and P. Zhu, "Hopf bifurcation in a fractional diffusion food-limited models with feedback control", Journal of Mathematical Chemistry, vol. 53, (2015), pp. 1393-1411.

[9] T. Yang and X. Chen, "Local L2 gain of Hopf Bifurcation Stabilization", Proceedings of the 47th IEEE Conference on Decision and Control, (2008); Cancun, Mexico.

[10] H. Zhao and W. Xie, "Hopf bifurcation for a small-world network model with parameters delay feedback control”, Nonlinear Dynamics, vol. 63, no. 3, (2011), pp. 345-357.

[11] A. Lerm, "Control of Hopf bifurcation in multi-area power systems via a secondary voltage regulation scheme”, IEEE Power Engineering Society Summer Meeting, (2002); Chicago, USA.

[12] G. Chen, K. Yap and J. Lu, "Feedback Control of Hopf bifurcations", IEEE International Symposium on Circuits \& Systems, vol. 3, no. 3, (1998), pp. 639-642.

[13] L. Ding and C. Hou, "Stabilizing control of Hopf bifurcation in the Hodgkin-Huxley model via washout filter with linear control term", Nonlinear Dynamics, vol. 60, no. 1, (2010), pp. 131-139.

[14] G. Itovich and J. Moiola, "Double Hopf Bifurcation Analysis Using Frequency Domain Methods", Nonlinear Dynamics, vol. 39, no. 3, (2005), pp. 235-258.

[15] F. Verduzco, J. Alvarez and A. Carrillo, "Normal Form and Control of the Hopf bifurcation", Proceedings of the 2007 American Control Conference, (2007); New York City, USA.

[16] R. Zhang, J. Li and G. Sun, "Hopf Bifurcation Control of Lü System via Washout Filter", Chinese Control and Decision Conference (CCDC), (2011); Mianyang, China.

[17] G. Duan, "Parametric Control Systems Design - Theory and Applications", International Joint Conference SICE-ICASE, Bexco, (2006); Busan, Korea.

[18] Y. Gorrec and C. Chiappa, "Controller Parametric Robustification Using Observer-Based Formulation and Multimodel Design Technique", IEEE Transactions on Automatic Control, vol. 50, no. 4, (2005), pp. 526-531.

[19] Z. Wu, R. Ding and Y. Chen, "Classification of parametrically constrained bifurcations", Applied Mathematics and Mechanics, vol. 31, no. 2, (2010), pp. 135-142. 
[20] Z. Wu and P. Yu, "A method for Stability and bifurcation control", IEEE Transactions on Automatic Control, vol. 51, no. 6, (2006), pp. 1019-1023.

[21] B. Demirel and L. Guvenc, "Parameter Space Design of Repetitive Controllers for Satisfying a Robust Performance Requirement", IEEE Transactions on Automatic Control, vol. 55, no. 8, (2014), pp. 18931899.

[22] S. LaValle, "Planning Algorithms, Chapter 6: Combinatorial Motion Planning", Cambridge University Press, (2006).

[23] H. Hjalmarsson and F. Egebrand, "Input design using cylindrical algebraic decomposition", The 50th IEEE Conference on Decision and Control and European Control Conference (CDC-ECC), (2011); Orlando, FL, USA.
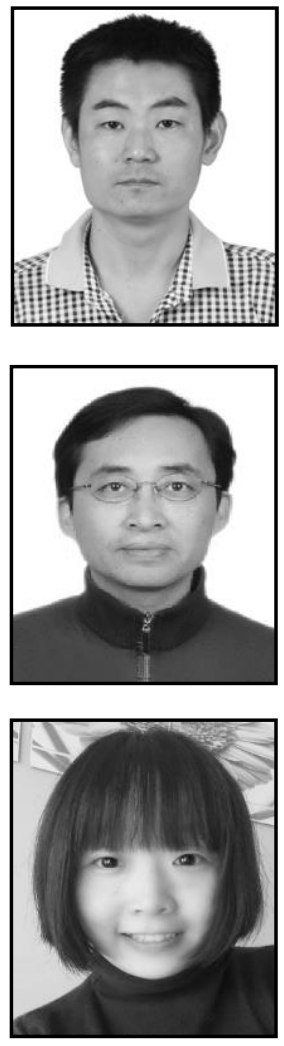

\section{Authors}

Jinbo Lu, He was born in Hebei Province, China, in 1978. He received his M.S. degree from school of electrical engineering, Yanshan University, Qinhuangdao, in 2004. He is currently pursuing the Ph.D. degree with School of Energy Science and Engineering, University of Electronic Science and Technology of China. His research interests include nonlinear control, intelligent control, and parametric controller design. E-mail: 564276721@qq.com.

Xiaorong Hou, He was born in Shanxi Province, China, in 1966. Currently, he is a professor of School of Energy Science and Engineering at the University of Electronic Science and Technology of China. He has published over eighty research papers and two monographs. His research interests include symbolic computation, real algebraic geometry, control theory, and intelligent systems. Email: houxr@uestc.edu.cn.

Min Luo, She received the B.S. degree in Testing and Measuring Technology and Instrumentation from Southwest Petroleum University, Chengdu in 2009. She is presently working toward the $\mathrm{Ph} . \mathrm{D}$. degree at the School of Energy Science and Engineering, University of Electronic Science and Technology of China. Her current research interests include nonlinear control, intelligent control, and applications. E-mail: 36205007@qq.com 
International Journal of Control and Automation Vol.10, No.3 (2017) 